[16] -, Uniformly non-square Orlicz spaces, Nieuw. Arch. Wisk. 14 (1966), 31-39.

[17] -, On the strict and uniform convexity of certain Banach spaces, Pacific J. Math. 15 (1965), 1083-1086.

[18] B. Turett, Rotundity of Orlicz spaces, Proc. Konink. Nederl. Akad. Wet. Amsterdam A 79.5 (1976), 462-468.

\section{Invariant states for positive operator semigroups}

by

\section{KLAUS E. THOMSEN (Aarhus)}

Abstract. A characterization is given of the semigroups of normal positive contractive operators on a von Neumann algebra that admit a faithful family of normal states invariant under the action of the semigroup. It is shown that the family (when it exists) may be chosen to consist of one single state if the algebra is $\sigma$-finite.

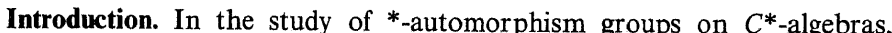
invariant states play an important role. Especially the existence of a faithful invariant state or set of states has been a useful tool in the investigation of such groups. In more recent years the existence of a faithful invariant state has been assumed also in connection with positive operator semigroups (see e.g. [6], [7], [15]). Therefore the question arises as to which positive semigroups of operators actually admit a single invariant faithful state, or at least a faithful family of invariant states. In 1972 St $\varnothing$ rmer [16] characterized the automorphism groups with a faithful family of invariant normal states on a von Neumann algebra. In this paper we will do the same for positive operator semigroups.

In fact we shall give three conditions which are equivalent to the existence of a faithful family of normal invariant states. Since we prove that such a family. (if it exists) can be chosen orthogonal, it follows that we may assume that the family consists of one state only if the algebra is $\sigma$-finite. If the algebra is not $\sigma$-finite, no faithful normal state can exist. Our results generalize and improve results in [11], [12] and [16].

For the readers not especially interested in semigroups, we remark that our results apply to normal positive contractive operators as well. If namely $\pi$ is such an operator, $\left\{\pi^{n} \mid n \in \boldsymbol{N}\right\}$ is a semigroup of the form considered in this paper.

We first recall some definitions and fix the notation.

(1) Let $\mathscr{M}$ be a von Neumann algebra acting on the Hilbert space $\mathscr{H}$ and $\mathscr{S}$ a semigroup of positive normal contractive operators on $\mathscr{M}$. By $\mathscr{L}(\mathscr{M})$ we denote the space of bounded operators on $\mathscr{M}$ and by $\mathscr{L}_{*}(\mathscr{M})$ the subspace of $\mathscr{L}(\mathscr{M})$ consisting of normal operators. A bar ${ }^{-}$will denote the closure in the point-weak topology of $\mathscr{L}(\mathscr{M})$. By [10] the unit ball $\mathscr{L}(\mathscr{M})_{1}$ is compact in this topology. 
(2) $\mathscr{S}$ is called ultraweakly almost periodic if it satisfies the following equivalent conditions:

(i) $\{\omega \circ S \mid S \in \mathscr{S}\}$ is relatively compact in the weak topology of $\mathscr{M}_{*}$ for each $\omega \in \mathscr{M}_{*}$,

(ii) $\overline{\mathscr{S}} \subseteq \mathscr{L}_{*}(\mathscr{M})$,

(iii) $\mathscr{S}$ is equicontinuous in the Mackey topology $\tau\left(\mathscr{M}, \mathscr{M}_{*}\right)$

(see [11] and [12]). By [3, V.6.4] the convex hull co $\mathscr{S}$ of $\mathscr{S}$ is ultraweakly almost periodic if $\mathscr{S}$ is.

(3) $\mathscr{S}$ is called weak ${ }^{*}$ mean ergodic if $\overline{\operatorname{co} \mathscr{S}}$ contains a normal projection $P$ such that

$$
S P=P S=P \quad \text { for all } S \in \mathscr{S} .
$$

$P$ is uniquely determined by these conditions and is a projection onto the fixpoint space $\mathscr{M}_{\mathscr{S}}$ of $\mathscr{S}$.

(4) $\mathscr{S}$ is called amenable if the space $B(\mathscr{S})$ of bounded complex functions on $\mathscr{S}$ admits an invariant mean (see [8]).

(5) An operator $A \in \mathscr{L}(\mathscr{M})$ is faithful if the conditions $a \geqslant 0$ and $A a=0$ imply $a=0$. Correspondingly, a set of normal functionals $\Omega$ is a faithful family if $a \geqslant 0$ and $\omega(a)=0$ for all $\omega \in \Omega$ imply $a=0$.

(6) A $J W^{*}$-subalgebra $\mathscr{J}$ of $\mathscr{M}$ is an ultraweakly closed subspace containing the unit such that $a, b \in \mathscr{J}$ implies $a b+b a \in \mathscr{J}$. The selfadjoint part of $\mathscr{J}$ is a $J W$-algebra as studied by Topping in [18].

(7) A weight $\varphi$ on $\mathscr{M}$ will be called $\mathscr{S}$-invariant ( $\mathscr{S}$-subinvariant) if $\varphi \circ S$ $=\varphi(\varphi \circ S \leqslant \varphi)$ for all $S \in \mathscr{S}$. As each state is a finite weight, we can use the same terms for states. For a weight $\varphi$ on $\mathscr{M}$ we let $\mathscr{M}_{\varphi}^{+}$denote the set $\left\{a \in \mathscr{M}^{+} \mid \varphi(a)<\infty\right\}$. If $\mathscr{J} \subseteq \mathscr{M}$ is a $J W^{*}$-subalgebra of $\mathscr{M}$, we say $\varphi$ acts semi-finitely on $\mathscr{J}$ if $\mathscr{M}_{\varphi}^{+} \cap \mathscr{J}$ is ultraweakly dense in the positive cone of $\mathscr{J}$. For further facts on weights we refer the reader to [9] and [14].

(8) Finally, the letter $N$ will denote the set of normal states of $\mathscr{M}$, and for $\omega \in N$ we let $\varrho_{\omega}$ denote the support projection of $\omega$.

Lemma 1. Let $\omega \in N$ be $\mathscr{S}$-invariant. Then

(i) $S\left(\varrho_{\omega}\right) \geqslant \varrho_{\omega}$ for all $S \in \mathscr{S}$.

If $\mathscr{S}$ admits a faithful $\mathscr{S}$-invariant semi-finite normal weight $\varphi$, then

(ii) $S(b a b)=b S(a) b$ for all $S \in \mathscr{S}, a \in \mathscr{M}, b \in \mathscr{M}_{\varphi}^{+} \cap \mathscr{M}_{\mathscr{S}}$.

If $\mathscr{S}$ admits a faithful family of normal $\mathscr{S}$-invariant states, then

(iii) $S\left(\varrho_{\omega}\right)=\varrho_{\omega}$ for all $S \in \mathscr{S}$.

Proof. (i) Clearly, $0 \leqslant S\left(\varrho_{\omega}\right) \leqslant I$. But $\omega\left(I-S\left(\varrho_{\omega}\right)\right)=0$, so $I-S\left(\varrho_{\omega}\right)$ $\leqslant I-\varrho_{\omega}$ which is (i).

(ii) We may assume $a=a^{*}$. Since $S\left(b^{2}\right) \geqslant S(b)^{2}=b^{2}$ by the generalized Schwarz inequality and $\varphi\left(S\left(b^{2}\right)\right)=\varphi\left(b^{2}\right)<\infty$, it follows that $S\left(b^{2}\right)=b^{2}$.
For $\mu \in N$ we define a positive bilineàr form on $\mathscr{M}^{\text {sa. }}$ by

$$
(c, d)=\mu(S(c \circ d)-S(c) \circ S(d)),
$$

where $c \circ d=\frac{1}{2}(c d+d c)$ for $c, d \in \mathscr{M}^{\text {sa. }}$. By using the Cauchy-Schwarz inequality for each such form, it follows that

$$
S(c \circ b)=S(c) \circ b \quad \text { for all } c \in \mathscr{M}^{\text {s.a. }} .
$$

Since $b a b=2 b \circ(a \circ b)-a \circ b^{2}$, the proof of (ii) is complete. $\mu \in N$.

(iii) follows easily from (i) since $\mu\left(S\left(\varrho_{\omega}\right)\right)=\mu\left(\varrho_{\omega}\right)$ for each $\mathscr{S}$-invariant

The next lemma is only a slight improvement of [12, Lemma 4.1].

LeMma 2. Assume $\mathscr{S}$ admits a faithful family $\Omega$ of normal states satisfying

$$
\omega(S(a) S(a)) \leqslant \omega\left(a^{2}\right), \quad S \in \mathscr{S}, \quad a \in \mathscr{M}^{\text {s.a. }}, \omega \in \Omega .
$$

Then $\mathscr{S}$ is ultraweakly almost periodic.

Proof. The proof of [11, Proposition 3.2] can be taken over word by word.

Proposition 3. Assume $\Omega \subseteq N$ is a faithful family such that

(*) $\quad \omega(S(a) S(a)) \leqslant \omega\left(a^{2}\right) \quad$ when $\omega \in \Omega, S \in \mathscr{S}$ and $a \in \mathscr{M}^{\text {s.a. }}$.

Then there exists a real Hilbert space $\mathscr{H}$ and an affine injective semigroup homomorphism $J: \overline{\operatorname{co\mathscr {S}}} \rightarrow \mathscr{B}(\mathscr{H})_{1} . J(\overline{\operatorname{co} \mathscr{S}})$ is strongly closed and if $\overline{\operatorname{co} \mathscr{S}}$ has the point-strong topology, $J$ is a homeomorphism from $\overline{\operatorname{co} \mathscr{S}}$ to $J(\overline{\operatorname{co} \mathscr{S}})$ with the strong topology.

Proof. Note first that the Cauchy-Schwarz inequality implies that $(*)$ holds on $\operatorname{co} \mathscr{S}$ as well as on $\mathscr{S}$. Since $\overline{\operatorname{co} \mathscr{S}}$ agrees with the point-strong closure of co $\mathscr{S}$, we have $(*)$ on all of $\overline{\operatorname{co} \mathscr{S}}$.

Let $\mathscr{H}_{\omega}, \omega \in \Omega$, be the real Hilbert space obtained by completing $\mathscr{M}^{\text {sa }} \varrho_{\omega}$ relative to the inner product $\langle\cdot, \cdot\rangle_{\omega}$ defined by

$$
\left\langle a \varrho_{\omega}, b \varrho_{\omega}\right\rangle=\operatorname{Re} \omega(a b) \text {. }
$$

To each $S \in \overline{\operatorname{co} \mathscr{S}}$ we can associate an operator $S_{\omega}$ on $\mathscr{H}_{\omega}$ by $S_{\omega}\left(a \varrho_{\omega}\right)$ $=S(a) \varrho_{\omega}$. By $(*), S_{\omega}$ is well-defined and extends to a contractive operator on $\mathscr{H}_{\omega}$. Now put

$$
\mathscr{H}=\sum_{\omega \in \Omega} \oplus \mathscr{H}_{\omega} \quad \text { and } \quad J(S)=\sum_{\omega \in \Omega} \oplus S_{\omega} .
$$

By construction $J$ defines an affine semigroup homomorphism into $\mathscr{B}(\mathscr{H})_{1}$. Since 1.u.b. $\varrho_{\omega}=I, J$ is injective. It follows from the definition of $J$ that $\omega \in \Omega$

$$
\left\langle J(S) a \varrho_{\omega}, b \varrho_{\omega}\right\rangle=\operatorname{Re} \omega(S(a) b) \quad \text { for } a, b \in \mathscr{M}^{\text {s.a. }}, \omega \in \Omega .
$$


From this it follows that $J$ is point-weak/weak continuous. Since $\overline{\operatorname{co} \mathscr{S}}$ is point-weakly compact, $J(\overline{\operatorname{co} \mathscr{S}})$ is weakly hence strongly closed. The homeomorphic nature of $J$ follows from [11, (2.2) Lemma].

THEOREM 4. The following conditions on $\mathscr{S}$ are equivalent:

(1) $\mathscr{S}$ admits a faithful family of $\mathscr{S}$-invariant normal states.

(2) $\mathscr{S}$ is weak* mean ergodic and the corresponding ergodic projection $P$ is faithful.

(3) (a) $\mathscr{S}$ is ultraweakly almost periodic,

(b) $\overline{\operatorname{co} \mathscr{S}}$ is amenable,

(c) $S(I)=I$ for all $S \in \mathscr{P}$,

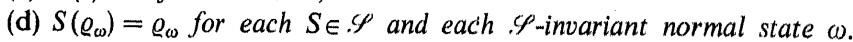

(4) $\mathscr{M}$ admits a faithful, normal semi-finite $\mathscr{S}$-invariant weight $\varphi$ acting semi-finitely on $\mathscr{M}_{\mathscr{S}}$.

If the conditions are satisfied, we can choose an orthogonal faithful family of $\mathscr{P}$-invariant normal states. $P$ is a positive unital normal projection onto the fixpoint space of $\mathscr{S}$ which is a $J W^{*}$-subalgebra of $\mathscr{M}$. Furthermore, $P$ satisfies $P(a b+b a)=P(a) b+b P(a)$ whenever $a \in \mathscr{M}$ and $b=P(b) \in \mathscr{M}$.

Proof. (1) $\Rightarrow(2)$. By Proposition 3 and the Alaoglu-Birkhoff mean ergodic theorem, $\operatorname{co} \mathscr{S}$ contains a projection $P$ satisfying $S P=P S=P$ for all $S \in \mathscr{S}$. By Lemma $2, P \in \mathscr{L}_{*}(\mathscr{M})$, so $\mathscr{S}$ is weak* mean ergodic.

If $a \geqslant 0$ and $P a=0$, then $\omega(a)=\omega(P a)=0$ for each $\mathscr{S}$-invariant normal state $\omega$. Therefore $a=0$ and we see that $P$ is faithful.

(2) $\Rightarrow(1) .\{\omega \circ P \mid \omega \in N\}$ is a faithful family of positive functionals, so after normalization we get (1).

(1) $\Rightarrow$ (3). (a) follows from Lemma 2.

By (2), $\mathscr{S}$ is weak ${ }^{*}$ mean ergodic, so evaluation at $P \in \overline{\operatorname{co} \mathscr{S}}$ defines an invariant mean on $B(\overline{\operatorname{co} \mathscr{S}})$. Thus (b) holds. If $\mu$ and $\omega$ are $\mathscr{S}$-invariant normal states, we have $\omega(I-S(I))=0$ and $\mu\left(S\left(\varrho_{\omega}\right)-\varrho_{\omega}\right)=0$. Thus (c) follows since $S(I) \leqslant I$; and (d) follows since $S\left(\varrho_{\omega}\right) \geqslant \varrho_{\omega}$ by Lemma 1 .

(3) $\Rightarrow$ (4). Let $\omega \in N$. By (a), $K=\{\omega \circ S \mid S \in \overline{\operatorname{co} \mathscr{S}}\}$ is a weak compact convex subset of $\mathscr{M}_{*}$. By (c) it contains only states.

Let $A(K)$ (respectively $A(\overline{\operatorname{co} \mathscr{S}})$ ) denote the weakly continuous affine functions on $K$ (respectively point-weak continuous affine functions on $\overline{\operatorname{co} \mathscr{\mathscr { P }}}$ ). Define $i: A(K) \rightarrow A(\overline{\operatorname{co} \mathscr{P}})$ by

$$
\text { if }(T)=f\left(T_{*} \omega\right), \quad T \in \overline{\operatorname{co} \mathscr{S}} .
$$

If $l$ is an invariant mean on $A(\overline{\operatorname{co} \mathscr{S}})$, then $l \circ i$ is a $\overline{\operatorname{co} \mathscr{S}}$-invariant state on $A(K)$. As is well-known, $l \circ i$ arises as evaluation at a point $\mu \in K$. This $\mu \in N$ is $\mathscr{P}$-invariant and agrees with $\omega$ on the fixpoint space of $\mathscr{S}$.

Now let $\Omega$ be a maximal set of orthogonal normal $\mathscr{S}$-invariant states.
Assume $\sum_{\omega \in \Omega} \varrho_{\omega}<I$ and put $q=I-\sum_{\omega \in \Omega} \varrho_{\omega}$. By (d), $S(q)=q$ for all $S \in \mathscr{S}$, i.e. $q$ is in the fixpoint space of $\mathscr{S}$. Choose $\omega \in N$ such that $\omega(q)=1$. As above we can find $\mu \in N$ such that $\mu$ is $\mathscr{S}$-invariant and $\mu(q)=\omega(q)=1$. Then $\varrho_{\mu} \leqslant q$, contradicting the maximality of $\Omega$. Thus $\sum_{\omega \in \Omega} \varrho_{\omega}=I$ and therefore $\Omega$ is a faithful family.

Put $\varphi(\cdot)=\sum_{\omega \in \Omega} \omega(\cdot)$. Then $\varphi$ is clearly a faithful normal $\mathscr{S}$-invariant weight. If $\left\{\omega_{1}, \ldots, \omega_{n}\right\} \subseteq \Omega$ is a finite set and $a \in \mathscr{M}^{+}$, then

$$
\varphi\left(\left[\sum_{i=1}^{n} \varrho_{\omega_{i}}\right] a\left[\sum_{i=1}^{n} \varrho_{\omega_{i}}\right]\right)=\sum_{i=1}^{n} \omega_{i}(a) \leqslant n\|a\|
$$

Since $\sum_{i=1}^{n} \varrho_{\omega_{i}} \in \mathscr{M}_{\mathscr{S}}$ and $\sum_{\omega \in \Omega} \varrho_{\omega}=I$, it follows that $\varphi$ is semi-finite on $\mathscr{M}$ and on $\mathscr{M}_{s \text {. }}$.

(4) $\Rightarrow$ (1). Since $I \in \mathscr{M}_{\mathscr{S}}$, we can find a net $\left\{a_{j}\right\} \subseteq \mathscr{M}_{\mathscr{S}} \cap \mathscr{M}_{\varphi}^{+}$such that $a_{j} \rightarrow I$ strongly. Define states $\omega_{j}$ on $\mathscr{M}$ by $\omega_{j}(\cdot)=\varphi\left(a_{j} \cdot a_{j}\right)$. The normality of $\varphi$ implies the normality of $\omega_{j}$, for all $j$ (see [9]). From Lemma 1 (ii) it follows that each $\omega_{j}$ is $\mathscr{S}$-invariant.

If $b \geqslant 0$ and $\omega_{j}(b)=0$ for all $j, a_{j} b a_{j}=0$ for all $j$ and thus $b=0$. Normalizing each $\omega_{j}$, we obtain a faithful family of $\mathscr{S}$-invariant normal states.

To complete the proof we only need to show that $P(a b+b a)=P(a) \times$ $\times b+b P(a)$ whenever $a \in \mathscr{M}$ and $b=P(b) \in \mathscr{M}$. But this follows by the argument that proved Lemma 1 (ii).

Remarks. (i) If $\mathscr{S}$ is a group of *automorphisms, (c) and (d) are trivially satisfied. Furthermore, in this case (b) follows from (a) (see [13]). Thus the theorem generalizes a result of St $\phi$ rmer in [16].

Simple examples show that neither (c) nor (d) follows from the other conditions. In case $\mathscr{S}$ is amenable and thus especially if $\mathscr{S}$ is abelian, (b) is automatic. To see this, consider $A(\overline{\operatorname{co} \mathscr{S}})$ and let $m$ be an invariant mean on $B(\mathscr{S})$. Define $\tilde{m}$ on $A(\overline{(\operatorname{co} \mathscr{P}})$ by

$$
\tilde{m}(f)=m\left(\left.f\right|_{\mathscr{G}}\right), \quad f \in A(\overline{(\operatorname{co} \mathscr{P}}) .
$$

Then $\tilde{m}$ is an $\mathscr{S}$-invariant state on $A(\overline{\operatorname{co} \mathscr{P}})$. Since $\overline{\operatorname{co} \mathscr{S}} \subseteq \mathscr{L}(\mathscr{M})_{1}$ is compact in the point-weak topology, $\tilde{m}$ arises as evaluation at a point $P \in \overline{\operatorname{co} \mathscr{S}}$. This $P$ is an ergodic projection for $\mathscr{S}$ and therefore evaluation at $P$ is an invariant mean on $B(\overline{\operatorname{co} \mathscr{S}})$. Thus (b) is satisfied.

However, (b) is not redundant in general. To see this, put $\mathscr{M}=C \oplus C$, and put $\mathscr{S}=\left\{S_{1}, S_{2}\right\}$ where $S_{1}(a, b)=(a, a)$ and $S_{2}(a, b)=(b, b)$, $(a, b) \in C \oplus C$. Then $\mathscr{S}$ is a semigroup because $S_{1}^{2}=S_{2} S_{1}=S_{1}$ and 
$S_{2}^{2}=S_{1} S_{2}=S_{2}$. Moreover, $S_{1}$ and $S_{2}$ are positive and unit-preserving. However, $\mathscr{S}$ admits no invariant states. Thus $\mathscr{S}$ satisfies (a), (c) and (d) but not (b). The author thanks the referee for this example.

(ii) In case $\mathscr{S}=\left\{S_{t}\right\}_{t \geqslant 0}$ is a $C_{0}^{*}$-semigroup, we are only left with conditions (a), (c) and (d) of the theorem. Condition (a) simply says that $\left\{S_{t}\right\}_{t \geqslant 0}$ must be an equicontinuous family in the Mackey topology $\tau\left(\mathscr{M}, \mathscr{M}_{*}\right) . C_{0}^{*}$-semigroups with this extra continuity condition have been extensively studied earlier (see e.g. [1, Chapter 3$]$ ). In this case we can give the following explicit formulas for the ergodic projection. In fact

$$
P a=\text { strong- } \lim _{\lambda \rightarrow 0} \lambda \int_{0}^{\infty} e^{-\lambda t} S_{t}(a) d t=\text { strong- } \lim _{\lambda \rightarrow \infty} \lambda^{-1} \int_{0}^{\lambda} S_{t}(a) d t \quad \text { for } a \in \mathscr{M} .
$$

This follows by combining [2, Theorem 5.1] with Proposition 3.

(iii) In applications it is often assumed that $\mathscr{S}$ consists of strongly positive operators, i.e. that

$$
S(a)^{*} S(a) \leqslant S\left(a^{*} a\right) \quad \text { for } S \in \mathscr{S}, a \in \mathscr{M} .
$$

One pleasant consequence of this is that $\mathscr{M}_{\mathscr{S}}$ is a ${ }^{*}$-subalgebra if $\mathscr{S}$ admits a faithful family of normal $\mathscr{S}$-invariant states. This was observed first by Frigerio [6]. Combining the arguments from [6] and a result from [17], it is seen that $\mathscr{M}_{\mathscr{S}}$ is a ${ }^{*}$-subalgebra if and only if $P$ is strongly positive. In this case $P$ is actually completely positive (see [4]).

It was shown in [5] that the range of a faithful positive projection in a $C^{*}$-algebra is always a Jordan subalgebra. Hence the Jordan structure of $\mathscr{M}_{\mathscr{S}}$ follows from the fact alone that it is the range of such a projection.

Corollary 5. $\mathscr{S}$ admits a faithful $\mathscr{S}$-invariant normal state if and only if $\mathscr{M}$ is $\sigma$-finite and $\mathscr{S}$ satisfies one of the equivalent conditions of the theorem.

Proof. The necessity is clear since a $W^{*}$-algebra admits a faithful normal state if and only if it is $\sigma$-finite. If $\mathscr{M}$ is $\sigma$-finite and $\mathscr{S}$ admits a faithful family $\Omega$ of normal $\mathscr{S}$-invariant states, we may assume, by the theorem, that $\Omega$ is countable. A suitable convex combination of the elements of $\Omega$ gives a normal faithful $\mathscr{S}$-invariant state.

Acknowledgement. The author gratefully acknowledges fruitful conversation on the subject of this paper with professors Erling St $\phi$ rmer, J $\phi$ rgen Vesterstr $\phi \mathrm{m}$ and Tage Bai Andersen.

\section{References}

[1] O. Bratteli and D. W. Robinson, Operator Algebras and Quantum Statistical Mechanics, Vol. I, Springer, Berlin-Heidelberg-New York 1979.

[2] E. B. Davies, One-parameter Semigroups, Academic Press, London etc. 1980

[3] N. Dunford and J. Schwartz, Linear Operators, part I, Interscience, New York 1958.
[4] E. G. Effros and E. C. Lance, Tensor products of operator algebras, Adv. in Math. 25 (1977), 1-34.

[5] - and E. St $\phi \mathrm{rmer}$, Positive projections and Jordan structure in operator algebras, Math Scand. 45 (1979), 127-138.

[6] A. Frigerio, Quantum dynamical semigroups and approach to equilibrium, Lett. Math Phys. 2 (1977), 79-87.

[7] -, Stationary states of quantum dynamical semigroups, Comm. Math. Phys. 63 (1978) 269-276.

[8] F. P. Greenleaf, Invariant means on topological groups and their applications, Van Nostrand, New York etc. 1969.

[9] U. Ha agerup, Normal weights on $W^{*}$-algebras, J. Funct. Anal. 19 (1975), 302-317.

[10] R. V. Kadison, The trace in finite operator algebras, Proc. Amer. Math. Soc. 12 (1961), 973-977.

[11] B. Kümmerer and R. Nagel, Mean ergodic semigroups on $W^{*}$-algebras, Acta Sci. Math (Szeged) 41 (1979), 151-159.

[12] A. T. Lau, W*-algebras and invariant functionals, Studia Math. 56 (1976), 253-261.

[13] R. Nagel, Mittelergodische Halbgruppen linearer Operatoren, Ann. Inst. Fourier (Grenoble) 23 (4) (1973), 75-87.

[14] G. K. Pedersen and M. Takesaki, The Radon-Nikodym theorem for von Neumann algebras, Acta Math. 130 (1973), 53-87.

[15] D. W. Robinson, Strongly positive semigroups and faithful invariant states, Comm. Math. Phys. 85 (1982), 129-142.

[16] E. St $\phi \mathrm{rmer}$, Invariant states on von Neumann algebras, Math. Scand. 30 (1972), 253-256.

[17] J. Tomiyama, On the projections of norm one in $W^{*}$-algebras, Proc. Japan Acad. 33 (1957), 608-612

[18] D. To pping, Jordan algebras of selfadjoint operators, Mem. Amer. Math. Soc. 53 (1965).

MATHEMATICS INSTITUTE, AARHUS UNIVERSITY Ny Munkegade, DK- 8000 Aarhus C, Denmark 